\title{
Research on Combination of Controllable Reactor and Magnetic Saturation Transformer
}

\author{
Zhiyou Ren ${ }^{1}$, Yan Zhao ${ }^{1}$, Xiaoyu Sun ${ }^{1}$, Yue $\operatorname{Han}^{2}$ and Xin $\operatorname{Sun}^{2}$ \\ ${ }^{1}$ Shenyang Institute of Engineering, Shenyang, China \\ ${ }^{2}$ Electric Power Research Institute, State Grid Liaoning Electric Power Supply \\ Co. Ltd., Shenyang China \\ *Corresponding author: zhaoyan@sie.edu.cn
}

\begin{abstract}
With intermittent energy grid connected, the requirements of transformer and reactive power compensation are developed. A novel combination technique of controllable reactor and magnetic saturation transformer is proposed in this paper. The optimal control target is implemented by combining the advantages of controllable reactor and magnetic saturation type transformer to suppress flicker of voltage effectively. The corresponding mathematical representation and the equivalent circuit are presented. The design and realization approach of this kind of transformer is described. The traditional transformer capacity can be promoted effectively.
\end{abstract}

Keywords: magnetic saturation transformer, controllable reactor, reactive power compensation, voltage control

\section{Introduction}

The extensive use of wind energy, solar energy and other large-scale intermittent power supply brings some difficulties of the grid control and safe operation. The main performance is one of the reactive power and the frequent changes greatly. It not only causes voltage drop in quality, but also brings great hidden danger to the security and stability of power system. In the background, it is a pressing matter of the moment of to seek the effective control of voltage and the compensation of reactive power while wind power, solar energy and other units connected to grid.

With the continuous expansion of grid scale and reactive power consumption of various electrical equipments, the problems of lacking of reactive power and voltage fluctuation are increasingly prominent. With intermittent energy grid connected, the requirements of transformer and reactive power compensation equipment technical are increased day by day [1-6]. Reference [3] proposes a new kind of hybrid genetic algorithm, which is composed of simple genetic algorithm and simulated annealing. Simulated annealing method is used to renew individual character. So the diversity of group is increased and the local optimum can be avoided. It can enhance stability of wind obviously.

Reference [7] proposes PI control method for a controllable reactor meets the needs of voltage control in power systems while its implementation in projects is easy. References [8-9] propose mathematical model and physical model through the application of controllable reactor in power system and selection methods. These methods have played a certain role in control the voltage stability, but some methods have limitations and difficult to be achieved.

Static Var Compensator (SVC) is one of the most commonly used equipments for reactive power compensation. SVC has a significant role in improving the stability of system. One of the main functions is that stabilize of the system voltage. Traditional SVC has their defects respectively. For example, thyristor controlled reactor produces large 
harmonic and needs to add additional filter. Moreover, in the working, the thyristor needs to bear all of the working voltage and current. At present, the domestic and foreign still cannot manufacture high voltage grade SCR. In order to improve the ability of SCR to withstand high voltage, a lot of SCRs are used in series, but the driver and insulation conditions are hard to guarantee [10-11].

According to the problems caused by methods of the traditional reactive power compensation in the process of intermittent energy connected with grid, a novel combination technique of controllable reactor and magnetic saturation transformer is proposed in this paper. These problems contain large harmonic contents, big loss, poor power quality, high investment cost and large amount of electrical equipment maintenance. The traditional transformer and reactor are used independently, and that not only causes the large equipment investment, but also brings certain difficulty to the operation and maintenance work, and the control of voltage and reactive power is poor. However, these disadvantages can be avoided by the combination technique of controllable reactor and magnetic saturation transformer. Magnetic conductor and winding of the hybrid controllable magnetic saturation of the transformer are rational distribution to reach the purpose of reducing the power loss and effectively suppress harmonics. Taking the wind power as an example, in the process that combination technique of controllable reactor and magnetic saturation transformer is applied to intermittent power connected with grid, combination technique of controllable reactor and magnetic saturation transformer can track to the change of the power of wind turbines fast.

Finally the following functions can be achieved:

(1) Compensates the reactive power of power grid.

(2) Eliminates the voltage fluctuation and improves power quality.

(3) The short-circuit current is limited.

(4) The cost of equipment investment and operation are reduced.

(5) The maintenance quantity of electrical equipment is decreased.

(6) The transmission capacity of power grid is improved.

(7) The reliability of power supply is improved.

\section{Preliminaries}

\subsection{The Controllable Reactor}

It is well known that the reactor is important equipment that power system compensates capacitive reactive power and limits the overvoltage. The capacity of some controllable reactor can be adjusted automatically, can limit over-voltage, reactive power regulation and reach the requirements of stability of the system [12-13]. According to the different structure principles, the controllable reactor can be divided into two categories. They are DC controllable reactor and the AC controllable reactor. Magnetically controlled reactor belongs to the DC controllable reactor. The structure of magnetic control reactor is as shown in Figure 1.

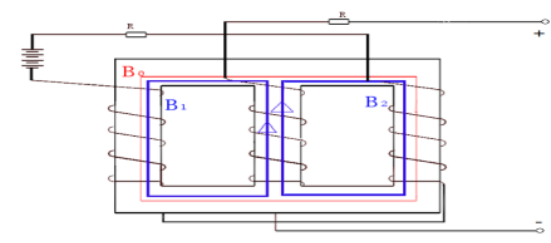




\section{Figure 1. Structure Diagram of Magnetic Control Reactor [14-19]}

The structure of magnetically controlled reactor is slightly complicated, but control is very simple. Magnetically controlled reactor is based on the magnetic saturation principle, so it can be adjusted continuously, but slow response speed and high harmonic content. But using appropriate structure and winding connection mode can restrict harmonic level in a certain range. AC controllable reactor is based on the principle of high impedance transformer. It can only classification regulation, not continuous smooth adjustment, but the fast response speed, low harmonic, small loss, and has obvious technical advantages [14-19]. This paper adopts the magnetic control reactor and magnetic saturation type transformer to constitute a hybrid controllable magnetic saturation transformer.

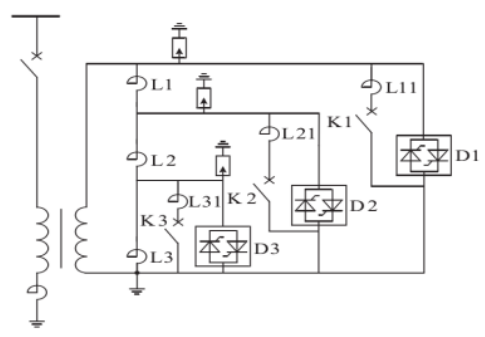

Figure 2. Schematic Diagram of Valve-Controlled Reactor [14]

Valve control type reactor is also called to magnetically controlled reactor (MCR). It works based on the principle of a magnetic amplifier. It is an iron core reactor of Ac / Dc magnetization at the same time and the saturation can be controlled. When it is working, the minimum DC power is used to change the operating point of control core. DC power is about $0.1 \% \sim 0.5 \%$ of the rated power of the reactor. Therefore, the inductance value is changed, so the purposes of regulating reactance current size and smooth adjustment of reactive power can be achieved [15-16].

\subsection{Magnetic Saturation Transformer}

Magnetic saturation phenomenon refers that when a coil through a current, there will be a magnetic field generated in the coil. There are two physical quantities for describing the magnetic field. One is the magnetic field intensity $\mathrm{H}$, which has a relationship with the product of coil turns and the size of current. The other is a magnetic induction intensity B, which has a relationship with the coil turn and medium of coil. When the medium is air, B is equal to $\mathrm{H}$. When the medium is the ferromagnetic material (such as iron, nickel), although the size of coil and current are same, the magnetic induction intensity B is far greater than the intensity of the magnetic field $\mathrm{H}$. The ratio value of $\mathrm{B}$ and $\mathrm{H}$ is a ferromagnetic material permeability $(\mu)$. The phenomenon that when the change of $\mathrm{H}$ is large but the change of $\mathrm{B}$ value is very small is known as phenomenon of magnetic saturation.

Magnetic saturation transformer is also called the constant voltage transformer. It has functions of AC stabilizer, anti-interference, transform voltage, over-current protection. And the structure is simple, economic, reliable, and easy to maintain. Magnetic saturation transformer has the functions of primary and secondary isolation, voltage transformation and multiplex voltage output. The most prominent characteristic of magnetic saturated transformer is that long-term short circuit will not burn. The disadvantages are the consistency of batch product is poor, the output frequency characteristic is poor, and the output waveform is close to a square wave, the high temperature, and big noise. 


\section{Combination of Controllable Reactor And Magnetic Saturation Transformer}

This paper presents a combination technique of controllable reactor and magnetic saturation transformer, and this concept of combination embodies in the following aspects: The first combination is the function of the controllable reactor and the function of transformer. The second combination is a variety of control winding, working and compensation winding and phase shift winding. The last combination is a reactor, a capacitor group, DC power and AC power. This paper proposes the new concept of 'combination' to solve many problems of intermittent power grid-connected operation effectively. These problems include the high harmonic caused by traditional ways of reactive power compensation, loss of large, poor power quality, high investment cost and large amount of electrical equipment maintenance. Because the response time of the magnetic valve controllable reactor is usually in the 15 power frequency cycle that is about $0.3 \mathrm{~s}$, which is far less than the requirements of suppressing flicker.

This method is proposed for the magnetic valve controllable reactor and transformer hybrid control strategy, and it has very high prospective and innovative. Schematic wiring diagram of the magnetic valve controllable reactor is as shown in Figure 2 [17].

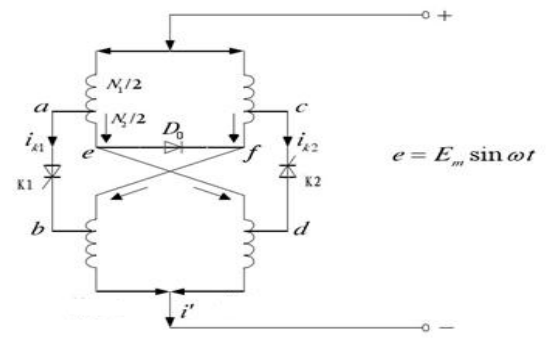

Figure 3. Operating Principle Figure of Magnetic Valve Controllable Reactor [17]

There are many characteristics of magnetic valve controllable reactor. It can change the voltage through the grid voltage and self winding, and obtain control power by thyristor components rectification. It does not need additional excitation. The working winding and control winding are organically combined together by the magnetic valve controllable reactor. This helps to reduce the power loss, and the structure is simplified [17].

The core section of the magnetic valve controllable reactor has a decrease. In the work scope of the reactor, only a small core section is saturated, and this is equal to a 'magnetic valve'. Due to the special structure of magnetic valve controllable reactor, therefore, it has smaller harmonic, the volt ampere characteristics of approximation linear, the smaller loss and fast response speed [17-18].

\section{The Working Principle and Structure of Hybrid Controllable Magnetic Saturation Transformer}

The novel combination technique integrates transformer, reactor, capacitors and other equipment. In fact, it is a special type of transformer. This transformer is equal to a reactive power. For the combination technique of controllable reactor and magnetic saturation transformer, there is a big difference from the conventional transformer and controllable reactor. These differences can be reflected in columnar iron core of transformer and its distribution of the winding. When the combination technique of controllable reactor and magnetic saturation transformer is applied to the wind farm 
reactive power voltage regulator, it is the transformer, and is also a controllable reactor at the same time.

In the process of reactive power compensation, the hybrid transformer and parallel capacitor group are used together. When the magnetizing current is zero, hybrid device works in the state of transformer. When the magnetizing current of hybrid device is a rated value, it works in the state of the controllable reactor. Under normal circumstance, the magnetizing current is between of zero and a rated value, so it has the function of transformer and controllable reactor.

For the winding distribution, the coil is hybrid winding. At the same time, it represents the primary winding of transformer and the reactor working winding are the secondary coil winding, and they reflect the function of transformer secondary winding, control winding and phase-shifting winding.

The whole device is composed of the three-phase same modules which connect with each other. Each phase has a magnetic conductor with two columns, and each conductor column was divided into two parts. The primary winding is composed of two coils, and the coil winding on the iron core 1 and 2, respectively. Then the coil connected with the AC power supply, produces circulatory flux in the magnetic conductor. Secondary winding consists of four branches, which are connected with four star structures respectively. One end is connected to the terminal $a, b, c$, and the other end is led out by the neutral point.

\section{The Equivalent Circuit and Its Equation of Hybrid Controllable Magnetic Saturation Transformer / Reactor}

For the combination technique of controllable reactor and magnetic saturation transformer, the equivalent circuit of the primary winding and secondary winding are as shown in Figure 4 and Figure 5.

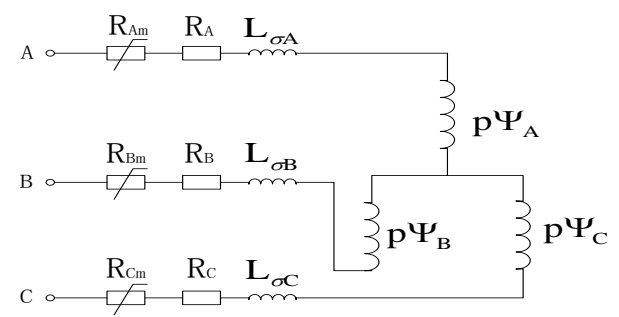

Figure 4. Equivalent Circuit Diagram of the Primary Winding

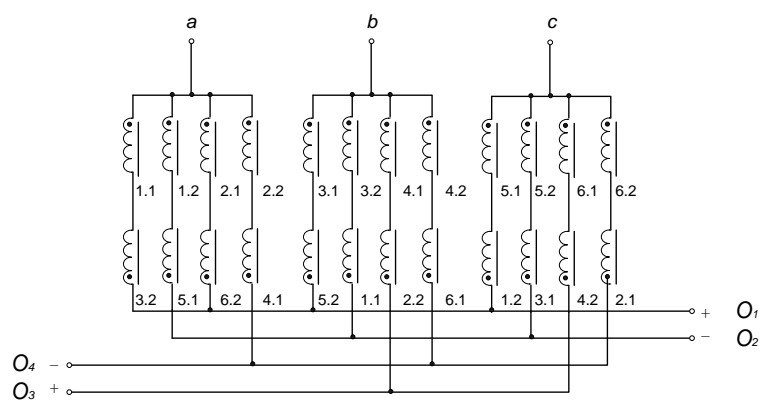

Figure 5. Equivalent Circuit Diagram of the Secondary Winding

Through the above analysis, and reference of Figure 4 and Figure 5, the equivalent circuit of primary winding is the same to the transformer. However, the equivalent circuit 
of the secondary winding is relatively more complicated. According to Kirchhoff's law and the equivalent circuit, the equation can be established as following:

$$
\left\{\begin{array}{l}
\mathrm{L}_{\sigma \mathrm{A}} p i_{\mathrm{A}}-\mathrm{L}_{\sigma \mathrm{B}} p i_{\mathrm{B}}+p \Psi_{\mathrm{A}}-p \Psi_{\mathrm{B}}=\mathrm{u}_{\mathrm{A}}-\mathrm{u}_{\mathrm{B}}-i_{\mathrm{A}}\left(\mathrm{R}_{\mathrm{A}}+\mathrm{R}_{\mathrm{A} m}\right)+i_{\mathrm{B}}\left(\mathrm{R}_{\mathrm{B}}+\mathrm{R}_{\mathrm{B} m}\right) \\
\mathrm{L}_{\sigma \mathrm{B}} p i_{\mathrm{B}}-\mathrm{L}_{\sigma \mathrm{C}} p i_{\mathrm{C}}+p \Psi_{\mathrm{B}}-p \Psi_{\mathrm{C}}=\mathrm{u}_{\mathrm{B}}-\mathrm{u}_{\mathrm{C}}-i_{\mathrm{B}}\left(\mathrm{R}_{\mathrm{B}}+\mathrm{R}_{\mathrm{B} m}\right)+i_{\mathrm{C}}\left(\mathrm{R}_{\mathrm{C}}+\mathrm{R}_{\mathrm{C} m}\right) \\
p i_{\mathrm{A}}+p i_{\mathrm{B}}+p i_{\mathrm{C}}=0
\end{array}\right.
$$

Further the corresponding equivalent magnetic circuit and the equation can be established, and the working state of controllable hybrid transformer is analyzed.

\section{Design and Implementation Approach}

The relevant parameters of the combination technique of controllable reactor and magnetic saturation transformer are calculated. First of all, the experiments of transformer no-load running must be done. Then the no-load current $I_{0}$, the ends voltage of the transformer $\left(U_{1}, U_{20}\right)$, and power loss $P_{0}$ are measured. Through these known quantities calculation of magnetizing impedance, ratio of transformer $\mathrm{K}$, and ferromagnetic loss $P_{F e}$. Because of no load current is very small, the copper loss generated by it is negligible. The main magnetic flux of transformer is greater than the leakage magnetic flux. Therefore, $Z_{m}>>Z_{1}$, no-load total impedance $Z_{0}=Z_{m}+Z_{1}$, and the load impedance is approximately equal to the excitation impedance [20].

Therefore, it can be concluded that the parameters of transformer

$$
\left\{\begin{array}{l}
Z_{m} \approx Z_{0}=\frac{U_{1 N}}{I_{0}} \\
R_{m}=\frac{P_{F e}}{I_{0}^{2}} \approx \frac{P_{0}}{I_{0}^{2}} \\
X_{m}=\sqrt{Z_{m}^{2}-R_{m}^{2}}
\end{array}\right.
$$

Secondly, through the short circuit experiment of transformer, the short circuit loss and short circuit impedance can be calculated. Due to the ferromagnetic loss is small, can be thought of input power is completely consumed on the copper loss. According to the experimental data $U_{K}, I_{K}, P_{K}$, the following formula can be calculated.

$$
\left\{\begin{array}{l}
Z_{k}=\frac{U_{K}}{I_{K}} \\
R_{K}=\frac{P_{C_{u}}}{I_{K}^{2}}=\frac{P_{K}}{I_{K}^{2}} \\
X_{K}=\sqrt{Z_{K}^{2}-R_{K}^{2}}
\end{array}\right.
$$

The above is the parameter formula of transformer that can be used to derive other formula [20-23].

Combination technique of controllable reactor and magnetic saturation transformer working mode can be divided into the following three types.

(1) Operational mode of the transformer rated load: 
In this manner of working, the magnetizing current is equal to 0 , and the field current $I_{m} \approx$ constant. At this time, combination technique is used as a transformer. It is working in rated current $\left(I_{1 N}, I_{2 N}\right)$ of primary winding and secondary winding.

(2) Reactor working state under the rated magnetizing current:

At this time, that is equivalent to no-load operations. As shown in Figure 5, terminals $\mathrm{a}, \mathrm{b}, \mathrm{c}$ are disconnected, and the secondary winding current $I_{2}=0$. The rated magnetizing current is dc $I_{N_{-}}$, that is to say under the rated voltage, magnetizing current is that rated current $I_{1 N}$ flows through primary winding.

(3) Hybrid working state:

At this time, electric current flows through the primary winding and $I_{1} \leq I_{1 N}$, primary winding current changes with the change of the load current and magnetizing current. At the same time, the field current is changing. Electric current flowing through the hybrid winding should not exceed the rated current of the transformer.

\section{Conclusion}

Through the above analysis and argumentation can draw that the combination technique of controllable reactor and magnetic saturation transformer is integrated with the functions of transformer and reactor. This technique has overcome the disadvantages of traditional method that large investment, maintenance difficulties and the poor of reactive voltage control. Through the reasonable distribution of magnetic conductor and the winding of controlled hybrid magnetic saturation of transformer, the purposes of reducing the voltage loss and harmonic suppression are reached.

However, the realization of combination technique of controllable reactor and magnetic saturation transformer is also faced with some technical difficulties. For example, the problems of intermittent power grid connected three-phase unbalance, the suppression of overvoltage in long distance transmission system, reduces the electromagnetic loss in the core and winding, and the control strategy of hybrid controllable magnetic saturation transformer.

It is gratifying that these problems have been solved, but has not yet reached the ideal state. Therefore, in the field of the intermittent energy grid connected and reactive power control, the combination technique of controllable reactor and magnetic saturation transformer has a very good development and application prospects.

\section{Acknowledgements}

This work is supported by National Nature Science Foundation of China under Grant 61304069, 61372195, 61371200 the Nature Science \& Foundation of Liaoning Province under Grant 2013020124, the Key Technologies R\&D Program of Liaoning Province under Grant 2012201010, the Key Project of Chinese Ministry of Education under Grant 212033, the Fundamental Research Fund of Liaoning Provincial Education Department Key Laboratory under Grant LZ2014050 and the Scientific Research Fund of Liaoning Provincial Education Department under Grant L2013494, L2012374.

\section{References}

[1] Y. L. Zhao, H. D. Li, L. Zhang and H. S. Zhang, "Wind Power Flow Optimization and Control System Based on Rapid Energy Storage", Proceedings of the CSEE, vol. 32, (2012), pp. 21.

[2] Z. H Bie, H. Liu, G. Li and X. F. Wang, "Voltage Fluctuation of a Power System with Wind Farms Integrated by Probabilistic Load Flow”, Journal of Xi'an Jiaotong University, vol. 42, (2008), pp. 1500. 
[3] X. L. Zhu, Y. Zhang, K. Gao, Q. Li, X. Z. Du and T. H. Liu, "Research on the Compensation of Reactive Power for Wind Farm", Power System Protection and Control, vol. 37, (2009), pp. 69.

[4] P. Xie, C. H. Peng and R. Yu, "Multi-objective Robust Optimized Scheduling of Power Grid Connected With Large-scale Intermittent Power Sources" Power System Technology, vol. 38, (2014), pp. 1480.

[5] W. J. Dong, X. M. Bai, N. H. Zhu, Z. G. Zhou and H. L. Li, "Discussion on the Power Quality Under Grid-connection of Intermittent Power Sources", Power System Technology, vol. 37, (2013) pp. 1265.

[6] C. F. Wang, J. Liang, L. Zhang, J. X. Feng and X. S. Han, "Optimization Decision Method of Wind Farm Reactive Power Compensation Capacity Considering Wind Power Distribution", Automation of Electric Power Systems, vol. 36, (2012), pp. 119.

[7] M. X. Tian, X. An, S. J. Gu and H. T. Chen, "Voltage-controlling Methods and Simulation Analysis of Magnetically Saturated and Transformer-type Controllable Reactors", High Voltage Engineering, vol. 39, (2013), pp. 791.

[8] M. X. Tian, Q. F. Li and S. H. Wang, "An Equivalent Physical Model and a Mathematical Model of the Controlled Saturable Reactor”, Transaction of China Electro technical Society, vol. 17, (2002), pp. 19.

[9] L. W. Zhou, Y. Xu, Q. Zhu and Y. H. Zhu, "Type Selection Analysis and Principle of New Controllable Reactor", Transformer, vol. 40, (2003), pp. 1.

[10] Q. T. Wu, J. Z. Wang, M. H. Zhao and Y. C. Ji, "A Novel Low Harmonic Content SVC and ITS Application", Automation of Electric Power Systems, vol. 27, (2003), pp. 58.

[11] J. Y. Wang, H. Q. Kong and G. W. Cai, "Magnetically-saturated Controllable Reactor Based Static Var Compensators", Electric Power, vol. 41, (2008), pp. 11.

[12] Q. Y. Zhou, Q. Guo, G. Q. Bu and L. G. Ban, “Application of Controllab1e Reactors in China's Power Grid at Extra and Ultra Voltage Level", Proceedings of the CSEE, vol. 27, (2007), pp. 1

[13] M. Liao and M. Ze, "Control Strategy and Protection Configuration for Step Controlled Shunt Reactor", Automation of Electric Power Systems, vol. 34, (2010), pp. 56.

[14] J. K. Zhang, X. H. Su, Y. Hu and D. X. Du, "Impact of Large-capacity Controllable Shunt Reactor on Line Differential Protection and Solutions", Automation of Electric Power Systems, vol. 38, (2014), pp. 115.

[15] J. H. Qian and B. C. Chen, "The Application of Reactive Power Compensator on Magnetite-valve Controllable Reactor in Electric Railway", Proceedings of the EPSA, vol. 15, (2003), pp. 65.

[16] X. X. Chen, C. H. Tian, B. C. Chen, Y. Z. Liu and Y. Jiaxin, "Mathematical Model for Harmonics Analysis of the Multi-Stage Saturable Magnetic-valve Controllable Reactor", Transactions of China Electro technical Society, vol. 26, (2011), pp. 57.

[17] C. S. Wang, W. X. Zhang and W. C. Fan, "Controlling Loop of Controllable Magnetic-valve Reactors and Its Characters", Electric Power Science and Engineering, vol. 4, (2005), pp. 46.

[18] J. B. Song, H. P. Wang, Z. Y. Zhang and G. S. Zhao, "Analytical Study of Controllable Reactor Based on Three-phase Magnetism Valve Type", Power System Protection and Control, vol. 37, (2009), pp. 20.

[19] J. X. Zhang, X. Wang, X. Lei and Z. F. Deng, "Overview of Controllable Reactor", Power System Technology, vol. 30, (2006), pp. 269.

[20] X. S. Han, "Principle and Design of Electronic Transformer", Railway Signaling \& Communication, vol. 45, (2009), pp. 24.

[21] Z. P. Wang, Y. Xu, X. Wang and Q. X. Yang, "Study on the Novel Transformer Protection Principle Based on the Transformer Model", Proceedings of the CSEE, vol. 23, (2003), pp. 54.

[22] W. J. Dai and Y. Chen, "Editor, Motor and Drive", Tsinghua University Press, (2008), Beijing.

[23] H. L. Zong, H. F. Jin, Z. F. Zhu and S. C. Zhang, "Transformer Inrush Detected by the Variation of Magnetizing Impedance", Proceedings of the CSEE, vol. 21, (2001), pp. 91.

\section{Authors}

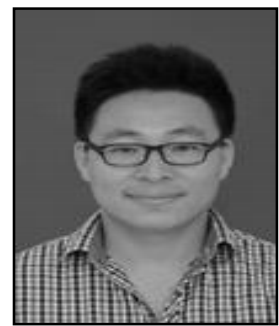

Zhiyou Ren, received the B.E. degree in Electrical Engineering and Automation from Liaoning Institute of Science and Technology, Benxi, China in 2013.

$\mathrm{He}$ is currently working towards the Master degree in Electric Engineering in Shenyang Institute of Engineering. 


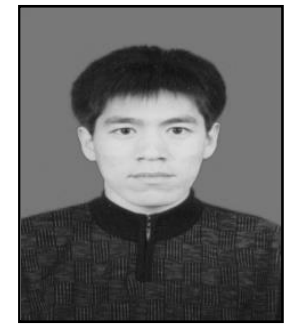

Yan Zhao, received the Ph.D. degree in Control Theory and Control Engineering from Northeastern University, Shenyang, China, in 2008.

$\mathrm{He}$ is currently an associate professor with the School of Renewable Energy, Shenyang Institute of Engineering, Shenyang. His current research interests include fuzzy control theory and distributed generation technique.

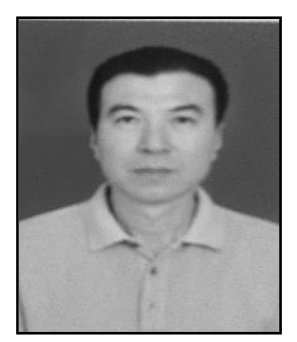

Xiaoyu Sun, He received the MBA degree from Liaoning University, mainly engaged in electrical device with intelligent controlling based on FPGA and soft core CPU.

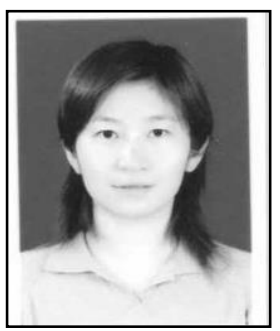

Yue Han, received the Master degree in Automation of Electrical Power System from North China Electric Power University, Beijing, China, in 2003. She is currently a senior engineer, working in Liaoning Electric Power Research Institute, Shenyang, China. Her current research interests include On-line Monitoring Technology and artificial intelligence.

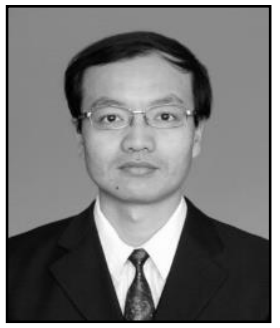

Xin Sun, was born in 1974. He received the master degree. He is currently a senior engineer with State Grid Electric Power Research Institute of Liaoning Provincial Power. His current research interest is power system relay protection. 
International Journal of Smart Home Vol. 9, No. 7 (2015) 